\title{
Contribution to the determination of the thermal emissivity of the composite material using long- wave infrared camera
}

\author{
Ondrej Stalmach ${ }^{1, *}$, Vladimir Dekys ${ }^{1}$, Frantisek Barnik ${ }^{1}$, Jan Simon $^{1}$, \\ ${ }^{1}$ University of Zilina, Faculty of Mechanical Engineering, Univerzitná 1, 010 26, Žilina, Slovakia
}

\begin{abstract}
This paper deals with measurement of emissivity of the composite samples. Three composite samples with the different thickness are heated to the temperature sixty-five degrees Celsius. On each sample must be applied a known emissivity coating. Unknown emissivity is calculated using the information obtained during the measurement. The aim of this paper is to show one of the possible approaches to find out the emissivity of the composite material.
\end{abstract}

Keywords: Thermography, thermogram, emissivity, composite, Onyx

\section{Introduction}

The world in which we live is characterized by features while, the most notable being the rapid frequency of differential innovation processes and the diversification of needs [1]. All sectors of the economy increase the proportion of the automation and robotics in their industries. More and more emphasis is placed on the complexity and complexity of the machines, while the reducing the number of production personnel. This results in increased requirements for operating parameters.

One of the key activities of the property care program is prevention. It consists of monitoring the status of monitored objects and diagnosing faults in the systems using the infrared thermography. Thermodiagnostics places a major emphasis on the area of temperature measurement by contactless and contacting methods. However, it is mainly aimed at displaying temperature fields, including its quantification. [1]

\section{Thermography}

Thermography or more accurate infrared thermography is a separate field of study that deals with contactless imaging, measurement, and temperature field analysis on the object surface. The role of thermography is the analysis of infrared energy that is radiated by the object. The infrared camera makes it possible to visualize the infrared radiation of an object that is not visible to the human eye, transforming it into electrical signals and displaying this radiation in the infrared camera. Thermography has proved to be a preventive maintenance tool for

\footnotetext{
* Corresponding author: Ondrej.Stalmach@fstroj.uniza.sk

Reviewers: Josef Soukup, Radim Halama
} 
tracking mechanical and electrical equipment, production processes, building objects, it is use in the field of science, research and development, but also in medicine.

\subsection{Definitions of the basic parameters}

Emissivity is defined as the ratio of the radiation intensity of the real body to the intensity of radiating an absolute black body with the same temperature. The emissivity of the absolute black body is 1 , the emissivity of the ideal mirror is 0 .

Ideal black body is a theoretical body that absorbs the total incident electromagnetic radiation from the surrounding of all wavelengths to its surface, with the same amount of energy emitting back to the environment.

Reflected apparent temperature is the apparent temperature of other objects that is reflected from the surface of the object to the infrared camera.

Thermogram is a temperature map or image of an object in which the distribution of the radiated energy from the surface of the object being measured is expressed by means of gray tinting or color imaging. [1]

\section{Basic principle of the thermography}

The basic principle of thermography is the detection of the radiation energy emitted from the measured object [1]. When measuring temperature by the infrared camera is measured the amount of energy that radiates or reflects the measured object to the measuring instrument. In this measurement, the emissivity of the material is important. Emissivity is a dimensionless quantity that determines how much heat the material radiates (emitting) to the environment. $[2,3]$

Figure 1 describes the interaction of different types of radiation with the object.

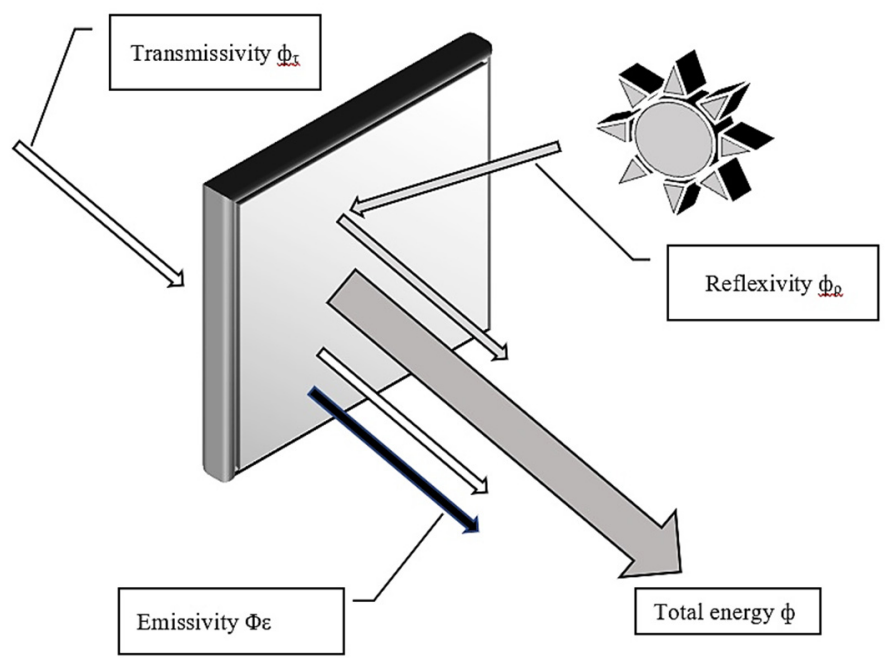

Fig. 1. Interaction of different types of radiation with the body

If an object is irradiated by the flux of $\Phi$, then part of this flow will be absorbed $\Phi \varepsilon$. Part of the flow will reflect $\Phi \rho$ a part of let $\Phi \tau$. The Energy Conservation Act Kirchhoff's law of thermal radiation applies:

$$
\Phi=\Phi \varepsilon+\Phi \rho+\Phi \tau=\varepsilon \Phi+\rho \Phi+\tau \Phi
$$


It follows that:

$$
\varepsilon+\rho+\tau=1
$$

$\varepsilon-$ Emissivity,

$\rho$ - reflexivity,

$\tau$ - transmissivity.

For the objects that are not transparent $\tau=0$, the following applies:

$$
\varepsilon+\rho=1
$$

In general, emissivity, reflectivity, and transmittance are the function of the wavelength, angle and temperature of the object. In our case, we are limited to wavelengths in the range $8-14 \mu \mathrm{m}, 10 \mathrm{deg}$ from normal to normal specimen area and $60^{\circ} \mathrm{C}$.

Modern composite materials are used as a substitute for classical materials. The production of structural elements made on $3 \mathrm{D}$ composite printers is relatively simple and inexpensive. It is therefore necessary to know the thermal emissivity of these materials to the thermal analysis. In our case this material is MarkForged's Onyx.

\section{Measurement of the reflected apparent temperature}

Reflected apparent temperature is the reflected radiation of objects around the measured object. To measure the reflected apparent temperature, the following conditions must be fulfilled:

- The calibrated infrared camera must allow to input the reflected apparent temperature and the emissivity.

- It is necessary to have a reflector that can be created by shrinking and re-aligning the shiny aluminium foil.

The procedure of the method for measuring the reflected apparent temperature is then as follows:

1. Set the emissivity value equal 1 on the infrared camera.

2. Position the camera so that the object is measured in the camera's field of view and that the camera is within the required distance from the measured object.

3. Position the reflector in front of the object being measured so that it is parallel to the surface of the object being measured.

4. Without changing the position, measure the temperature on the reflector using the camera, this temperature is the reflected apparent temperature (Trefl).

5. For the sake of greater precision, repeat this procedure from the 2 nd point to the 4 th point at least three times and then the average of the measured values.

6. The mean value of the reflected apparent temperature thus obtained is then entered directly into the camera or into the appropriate software. [1]

\section{Calculation of the emissivity from an available information}

Composite materials play a major role in the industry. Knowledge of emissivity in these materials is essential for quantitative thermography, [5-8]. Three composite samples of different thicknesses are used in the experiment. The material from which the samples are made using a 3D printer is named onyx. The material from which the samples are made using a 3D printer is named onyx. The long-wave infrared camera Flir E50 is used in the experiment. 
The first sample has a thickness of $0.4 \mathrm{~mm}$ and has $100 \%$ fill. The second sample has a thickness of $1 \mathrm{~mm}$ and has a $60 \%$ fill with a triangular structure. The last one has a thickness of $6 \mathrm{~mm}$ and has a $60 \%$ fill with a triangular structure.

On the samples must be applied a known emissivity coating. In the experiment are used $3 \mathrm{M}$ Scotch super 33+ PVC electrical insulation tape with the known emissivity $\varepsilon=0.95 \pm$ 0.05 and the ThermaSpot with known emissivity $\varepsilon=0.96 \pm 0.04$. The Thermaspot are applied on the all samples but the 3M Scotch super 33+ PVC electrical insulation tape are applied only on the first and second samples.

The procedure of the method for obtaining information which are important to calculate unknown emissivity of the composite samples as follows:

1. The electric heater is placed in a box made of polystyrene to eliminate the reflections from the environment.

2. The sample is placed on the electric heater and it is heated to the 65 degrees Celsius.

3. The reflector is placed in front of the composite sample so that it is parallel to the surface of the sample.

4. With the infrared camera Flir E50 the 100 snapshots are taken at $30 \mathrm{~Hz}$ scanning rate.

5. The reflector is removed, and another 100 snapshots are taken.

6. Steps 4. and 5. are repeated until do not have the 500 snapshots with the reflector and 500 without a reflector.

7. The obtained data is then processed in the software MATLAB.

8. Every snapshot can be uploaded to the MATLAB as a 2 D matrix with the size $240 \times 180$ because the infrared camera Flir E50 has a resolution of $240 * 180$ pixels.

9. From the snapshots are selected areas of interest representing the region of the known emissivity and the region with the unknown emissivity.

10. From these data a 3D matrix is created, and then its average value and standard deviation are calculated. These parameters are used to calculate the unknown emissivity value.

The figure 2 illustrates an example where the infrared camera records the radiations from the object on which a known emissivity coating is applied.

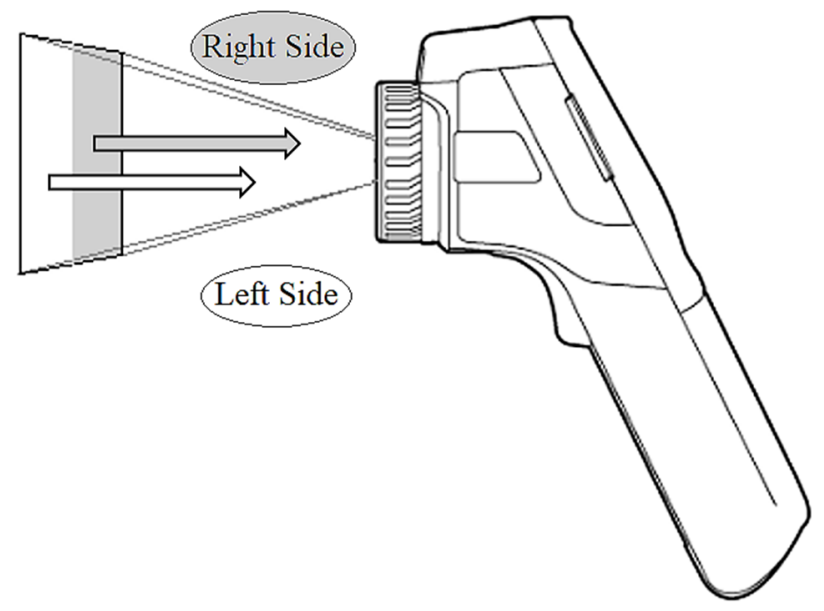

Fig. 2. Object with the regions of known and unknown emissivity.

Emissivity calculation is based on following formulas:

$$
\begin{aligned}
& T_{1}=\varepsilon_{1} \cdot T_{o b j}+\left(1-\varepsilon_{1}\right) \cdot T_{r e f l} \\
& T_{2}=\varepsilon_{2} \cdot T_{o b j}+\left(1-\varepsilon_{2}\right) \cdot T_{r e f l}
\end{aligned}
$$


$T_{1}$ - the temperature measured by the infrared camera on the surface of unknown emissivity

(Left side).

$T_{2}$ - the temperature measured by the infrared camera on the surface of known emissivity

(Right side),

$\varepsilon_{1}$ the unknown emissivity,

$\varepsilon_{2}$ - the known emissivity,

$T_{\text {refl }}$ - the reflected apparent temperature,

$T_{o b j}$ - the temperature of the object.

If the assumption is that the temperature at the surface of the object $\left(\mathrm{T}_{\mathrm{obj}}\right)$ is the same, the unknown emissivity $\varepsilon_{1}$ is calculated as follows:

$$
\varepsilon_{1}=\frac{\varepsilon_{2}\left(T_{1}-T_{r e f l}\right)}{\left(T_{2}-T_{r e f l}\right)}
$$

The value of the unknown emissivity is not a constant, but it is the value from the interval. To know this interval, it is necessary to calculate the standard deviation of the indirect variable $\left(\varepsilon_{1}\right)$ that depends on the multiple of variables $\left(T_{1}, T_{2}, T_{r e f l}, \varepsilon_{2}\right)$. The formula is as follows:

$$
\begin{aligned}
& \delta_{\varepsilon_{1}}=\sqrt{\left(\frac{\partial \varepsilon_{1}}{\partial \varepsilon_{2}}\right)^{2} \cdot \delta_{\varepsilon_{2}}{ }^{2}+\left(\frac{\partial \varepsilon_{1}}{\partial T_{1}}\right)^{2} \cdot \delta_{T_{1}}{ }^{2}+\left(\frac{\partial \varepsilon_{1}}{\partial T_{r e f l}}\right)^{2} \cdot \delta_{T_{r e f l}}{ }^{2}+\left(\frac{\partial \varepsilon_{1}}{\partial T_{2}}\right)^{2} \cdot \delta_{T_{2}}{ }^{2}} \\
& \delta_{\varepsilon_{1}}=\sqrt{\left(\frac{\left(T_{1}-T_{\text {refl } l}\right)}{\left(T_{2}-T_{\text {refl }}\right)}\right)^{2} \cdot \delta_{\varepsilon_{2}}{ }^{2}+\left(\frac{\varepsilon_{2}}{\left(T_{2}-T_{\text {refl }}\right)}\right)^{2} \cdot \delta_{T_{1}}{ }^{2}+}
\end{aligned}
$$

The results are shown in the Table 1.

Table 1. Results of the unknown emissivity

\begin{tabular}{|c|c|c|}
\hline & ThermaSpot & 3M Scotch super 33+ \\
\hline Sample 1 & $\varepsilon_{1}=0,91 \pm 0,04$ & $\varepsilon_{1}=0,91 \pm 0,05$ \\
\hline Sample 2 & $\varepsilon_{1}=0,93 \pm 0,04$ & $\varepsilon_{1}=0,94 \pm 0,05$ \\
\hline Sample 3 & $\varepsilon_{1}=0,94 \pm 0,05$ & - \\
\hline
\end{tabular}

\section{Conclusion}

The aim of this article is to show how to calculate the unknown emissivity of composite samples by the so-called scientific method and to indicate the values for Onyx material used by Markforged in their 3D printers. Knowledge of emissivity is required for the quantitative assessment of object temperatures.

It was found that the emissivity of the printed samples ranged from 0.91 to 0.94 . This interval is explained by the possible variability of print process parameters. Two-layer cover area was used on the sample. If identical print parameters are not followed, the assumption $\tau$ $=0$ may not be correct because in some cases the printed non-zero-transmitted surface may be printed. This case arises, for example, then the individual filaments have very small slits between them and the two-layer printed surface has very fine mesh properties. 
This contribution is a continuation of the computational work on composite materials, infrared camera measurements as well as non-destructive testing of materials performed at the Department of Applied Mechanics of the Faculty of Mechanical Engineering of the University of Zilina (Zilinska univerzita v Ziline), [9-14].

This paper was supported by VEGA 1/0795/16 and KEGA 017ŽU-4/2017 and by the Slovak Research and Development Agency under the contract No. APVV-0736-12.

\section{References}

1. V. Pet'ková, J. Svoboda, Thermodiagnostics. ISBN 978-80-8126-132-9 (2016)

2. M. Honner, P. Honnerova, Survey of emissivity measurement by radiometric methods. Applied Optics, 54, 669-683, (2015)

3. M. Honner, P. Litoš, M. Švantner, Thermography analyses of the hole-drilling residual stress measuring technique. Infrared Physics \& Technology, 45, 131-142, (2004)

4. P. Honnerova, J. Martan, M. Kucera, Martin, M. Honner, J. Hameury, New experimental device for high-temperature normal spectral emissivity measurements of coatings, Measurement Science and Technology, 25, Art.No. 095501 (2014)

5. P. Pastorek, P. Novak, P. Kopas, M. Mocilan, Finite element analysis of bond behavior in a steel reinforced concrete structure strengthened carbon fibre reinforced polymer (CFRP) strips. METALURGIJA, 56, 405-408, (2017).

6. Z. Murcinkova, P. Novak, V. Kompis, M. Zmindak, Homogenization of the finite-length fibre composite materials by boundary meshless type method. Archive Of Applied Mechanics, 88, 789-804, (2017)

7. P. Novak, M. Zmindak, Z. Pelagic, High-Pressure Pipelines Repaired by Steel Sleeve and Epoxy Composition. Applied Mechanics and Materials 1, 486, 181-188, (2014)

8. L. Radziszewski, M. Saga, Modeling of non-elastic properties of polymeric foams used in sports helmets. Procedia Engineering. 177, 314-317 (2017)

9. M. Kekez, L. Radziszewski, A. Sapietova, Fuel type recognition by classifiers developed with computational intelligence methods using combustion pressure data and the crankshaft angle at which heat release reaches its maximum Procedia Engineering, 136, 353-358, (2016)

10. R. Halama, P. Gál, P. Paška, Z. Sedlák, A new accelerated technique for validation of cyclic plasticity models. MATEC Web of ConferencesVolume, 157, Art. No. 05008, (2014)

11. I. Glodova, T. Liptak, J. Bocko, Usage of finite element method for motion and thermal analysis of a specific object in SolidWorks environment. Procedia Engineering, 96, 131135, (2014)

12. M. Handrik, M. Vasko, P. Kopas, V. Mozer, The linear and nonlinear stability loss of structures due to thermal load. Procedia Engineering. 136, 359-364 (2016)

13. Z. Murcinkova, P. Novak, V. Kompis, M. Zmindak, Homogenization of the finite-length fibre composite materials by boundary meshless type method. ARCHIVE OF APPLIED MECHANICS, 88, 789-804, (2017)

14. P. Novak, M. Zmindak, Z. Pelagic, High-Pressure Pipelines Repaired by Steel Sleeve and Epoxy Composition. Applied Mechanics and Materials1, 486, 181-188, (2014) 